ing (RLS) particle technology, a novel, ultra-sensitive and relatively simple signal generation and detection technology, to microarray systems. Submicroscopic RLS gold and silver particles of uniform dimension (20-120 nm diameter range) scatter incident white light to generate coloured light that appears as highly intense fluorescence. The intensity of coloured light signal generated by a single RLS particle is $10^{4}-10^{6}$ times greater than the signal from a single molecule of the most sensitive fluorophore. The intense RLS signal does not photobleach and the colour of scattered light generated by RLS particles depends on particle composition, size, shape and the refractive index of the scattering medium. The intensity and colour of individual RLS particles can be accurately predicted to develop multiplex detection systems by practical application of mathematical calculations derived from electromagnetic radiation theory ${ }^{1}$. The high level of signal intensity allows detection at extremely low particle concentration by the unaided eye $\left(10^{-15}-10^{-16} \mathrm{M}\right)$ and single RLS particles can be detected under appropriate illumination conditions using low-power magnification ${ }^{2}$. On solid surfaces, the RLS signal can be detected by the unaided eye at particle densities as low as $0.01-0.05$ particles per square micron. RLS particles can be derivatized with a variety of molecules for analytical bioassays including proteins, antibodies, small molecule ligands, nucleic acids and oligonucleotide probes. Once derivatized, RLS particles are highly selective for detection of specific biomolecular targets. Simple detection instrumentation consisting of an illumination source, configured light microscope, video camera and image analysis system has been developed and successfully applied to microarrays. The optical properties and practical application of RLS particles for microarray-based gene expression and DNA sequence analysis will be discussed.

1. Yguerabide, J. \& Yguerabide, E.E. Anal. Biochem. 262, 137-156 (1998).

2. Yguerabide, J. \& Yguerabide, E.E. Anal. Biochem. 262, 157-176 (1998).

\section{Pilarsky, Christian}

\section{Expression analysis using low amounts of mRNA and GeneChips}

\author{
Christian Pilarsky, Armin Schmitt, Simone Hille \\ \& André Rosenthal
}

metaGen GmbH, Ihnestr. 63, 14195 Berlin, Germany

Expression analysis could be used for the detection of novel regulatory networks and characterisation of primary tumours, leading to an improvement of cancer diagnosis. For this purpose metaGen has designed a custom GeneChip containing known tumour-associated genes (for example PTEN) and 1,500 cDNAs which were shown to be differentially expressed in EST libraries of several tumours by bioinformatics methods. Microdissected tissue needs to be used for accurate transcript profiling of solid tumours, but only low amounts of mRNA can be isolated from this source. Therefore validated and robust amplification techniques must be established. We compared the transcript profiles obtained from various amounts of mRNA as starting material from the human breast cancer cell line MDA-MB231. As a standard for comparison we used $300 \mathrm{ng}$ of poly(A)+RNA which was labelled according to the Affymetrix protocol. For the amplification of $10 \mathrm{ng}, 1$ ng, $100 \mathrm{pg}$ and $10 \mathrm{pg}$ we applied a descibed aRNA technique ${ }^{1}$ until $300 \mathrm{ng}$ of aRNA was obtained. This RNA was then labelled according to a modified Affymetrix protocol. For hybridisation and detection of the RNA we used the standard Affymetrix protocols. The GeneChips were analysed by the Affymetrix GeneChip software 3.1 and the coefficients for the average differences of the five experiments were calculated. For each of the four RNAs two cycles of preamplification with cDNA synthesis and in vitro transcription were necessary. We observed a correlation coefficient of 0.97 between the hybridisations with $10 \mathrm{ng}$ and $1 \mathrm{ng}$, whereas the correlation coefficient for the hybridisations with $10 \mathrm{ng}$ and $100 \mathrm{pg}$ was 0.81 . The correlation between the hybridsations with $300 \mathrm{ng}$ (no preamplification) and $10 \mathrm{ng}$ or $1 \mathrm{ng}$ was 0.79 and 0.77 , respectively. The correlation was weaker for the two other experiments. The preamplification of mRNA enables us to use low amounts of tissue. Based on the assumption that each cell contains roughly $0.5 \mathrm{pg}$ mRNA, only 2,000 cells are required per hybridisation experiment. For better reproducibility expression levels should only be compared with samples that have undergone the same number of preamplification cycles.

1. Luo, L. et al. Nature Med. 5, 117-122 (1999).

Pinkel, Daniel

\section{Technical approaches for efficient printing of high-density microarrays and rapid, high-precision fluorescence analysis}

\author{
D. Pinkel ${ }^{1}$, G. Hamilton ${ }^{1}$, A. Jones², D. Davy', M. Zom², \\ R. Segraves ${ }^{1}$, A. Snijders ${ }^{1}$, K. Livezey ${ }^{1}$, J. Gray ${ }^{1} \&$ D. Albertson ${ }^{1}$ \\ ${ }^{1}$ Cancer Genetics Program, University of California, San Francisco Cancer \\ Center, San Francisco, California, USA \\ ${ }^{2}$ E.O. Lawrence Berkeley National Laboratory, Berkeley, California, USA
}

We have developed an integrated microarray production and analysis facility for quantitative comparisons of DNA copy number and RNA expression. The overall process is managed by a custom Oracle database that tracks clone acquisition and preparation of printing solutions, manages array printing and image acquisition, and stores results of the fluorescence analysis. The printing robot employs printing pins made from quartz capillary tubing with an outside diameter of approximately $0.4 \mathrm{~mm}$, which has been heated and pulled to have an opening of approximately $25-50 \mu \mathrm{m}$. Each spot contains $50-100 \mathrm{pl}$ of solution. Maximum load of a pin is restricted to approximately $0.2 \mu 1$, permitting thousands of spots to be printed from one load, and numerous print runs to be made from the same plates. Standard source plates contain 864 wells, so that a 16-pin print head prints arrays that are $12 \mathrm{~mm}^{2}$. The small diameter of the pins will permit printing out of 1,536 well plates, so that 36 pins could be used to print $13.5-\mathrm{mm}$ arrays. Pin tip dimensions are being optimized to permit routine printing on less than $100-\mu \mathrm{m}$ centers. The overall goal is to keep the array size small to minimize the amount of specimen material required for analysis, while employing a large number of printing pins to increase printing rate. The array-imaging system uses a CCD camera, allowing acquisition of 16-bit fluorescence data from the entire array with an exposure of several seconds or less for each fluorochrome. The custom-designed imaging lenses are chromatically corrected from 450 to $750 \mathrm{~nm}$, and they provide flat images over the entire field. Illumination is supplied by a mercury arc lamp. Excitation and emission bands are established by computer-controlled filter wheels. Emission of at least four fluorochromes can be independently measured from a single specimen. These typically include DAPI, which is used as a counterstain to permit spot localization, and three labels in the specimen DNAs or RNAs, for example fluorescein, Cy3 and Cy5. Fluorescence ratios are calculated using custom software that automatically segments the spots of each sub array (the portion of the array printed by each pin) based on the DAPI image, determines local background and integrates signal intensities within each segmented spot. Our techniques permit measurement of genomic DNA copy number with ratio coefficient of variation of approximately 0.07 at the single-copy level, and measurement of expression levels with signal intensities approximately 100 -fold lower than -actin (ratio coefficient of variation $=0.1$ ). 